\title{
Investigation Into the Electromagnetic Impulses From Long- Pulse Laser Illuminating Solid Targets Inside a Laser Facility
}

\author{
Tao YI ${ }^{1}$, Jinwen YANG ${ }^{1,2}$, Ming YANG ${ }^{2}$, Chuanke WANG ${ }^{1}$, Weiming YANG ${ }^{1}$, \\ Tingshuai LI $^{2 *}$, Shenye LIU ${ }^{1}$, Shaoen JIANG ${ }^{1}$, Yongkun DING ${ }^{1}$, and \\ Shaoqiu XIAO ${ }^{3}$
}

\author{
${ }^{1}$ Laser Fusion Research Center, Chinese Academy of Engineering Physics, Mianyang, 621900, China \\ ${ }^{2}$ School of Energy Science and Engineering, University of Electronic Science and Technology of China, Chengdu, \\ 611731, China \\ ${ }^{3}$ School of Physical Electronics, University of Electronic Science and Technology of China, Chengdu, 611731, China \\ *Corresponding author: Tingshuai LI_Ｅ-mail: litingshuai@uestc.edu.cn
}

\begin{abstract}
Emission of the electromagnetic pulses (EMP) due to laser-target interaction in laser facility had been evaluated using a cone antenna in this work. The microwave in frequencies ranging from several hundreds of $\mathrm{MHz}$ to $2 \mathrm{GHz}$ was recorded when long-pulse lasers with several thousands of joules illuminated the solid targets, meanwhile the voltage signals from $1 \mathrm{~V}$ to $4 \mathrm{~V}$ were captured as functions of laser energy and backlight laser, where the corresponding electric field strengths were obtained by simulating the cone antenna in combination with conducting a mathematical process (Tiknohov Regularization with L curve). All the typical coupled voltage oscillations displayed multiple peaks and had duration of up to $80 \mathrm{~ns}$ before decaying into noise and mechanisms of the EMP generation was schematically interpreted in basis of the practical measuring environments. The resultant data were expected to offer basic know-how to achieve inertial confinement fusion.
\end{abstract}

Keywords: Electromagnetic impulse; laser; voltage; simulation; decay

Citation: Tao YI, Jinwen YANG, Ming YANG, Chuanke WANG, Weiming YANG, Tingshuai LI, et al., "Investigation Into the Electromagnetic Impulses From Long-Pulse Laser Illuminating Solid Targets Inside a Laser Facility,” Photonic Sensors, 2016, 6(3): $249-255$

\section{Introduction}

Although various radiations tend to be generated when some particles strike solid targets like $\mathrm{Cu}[1]$, the concentration of extremely powerful laser beams onto a solid target would induce significant electromagnetic radiation, which can not be only used for scientific research concerning the physics of extreme energy densities and pressures, but also provide a feasible path to achieve inertial confinement fusion (ICF) and a promising way to generate carbon-free sustainable energy [2]. Fast heating of ultrahigh-density plasma was reported to be an effective step towards laser fusion ignition [3]. However, an intensive electromagnetic pulse (EMP) emitted within the target chamber of a high power laser are well-known issues that cause malfunction and damage of some hardware, internal, and external diagnostics for the laser fusion. The EMP is possibly originated by the escaping electrons from the target surface during the laser-target interaction process [4], in which the amplitudes of EMP are closely related

Received: 20 May 2016 / Revised: 3 June 2016

(C) The Author(s) 2016. This article is published with open access at Springerlink.com

DOI: $10.1007 / \mathrm{s} 13320-016-0346-4$

Article type: Regular 
to the target sizes and laser power density. It is thus significantly imperative to evaluate the EMP inside and outside laser chamber before some necessary electromagnetic shielding fashions can be proposed to protect all the diagnostic setups.

Brown et al. [5] at Lawrence Livermore National Laboratory installed multiple B- and D-dot sensors in the Titan short-pulse laser facility (ps) to measure the levels of electromagnetic fields, by which the electric field strength from the sensors locating about $32 \mathrm{~cm}$ from target chamber center was estimated to be $167 \mathrm{kV} / \mathrm{m}$. During this process, the ps and ns laser pulses generated quite different ion emission current densities, leading to up to $100 \mathrm{MeV}$ by sub-picosecond laser plasma [6]. On the other hand, Brown et al. [7] also reported that the EMP signals collected at Lawrence Livermore National Laboratory mainly covered the frequency domains from several hundreds of $\mathrm{MHz}$ to several GHz. Chen et al. [8] reported the microwave emission generated by a laser pulse incident on a metallic disc surface had a frequency ranges from $0.5 \mathrm{GHz}$ to $4 \mathrm{GHz}$. Moreover, the fs laser pulse inducing EMP can be enhanced by a long (ns) laser pulse due to the formation of pre-plasma [9].

The generation of EMP by laser illuminating solid targets is closely bound up with the plasmas induced during this process. A long-pulse laser of high power density focuses on the solid target surface, rapidly producing plenty of plasma and the time needed is far shorter than that of the laser pulse. Moreover, for the long-pulse laser without high power density $\left(<10^{15} \mathrm{~W} / \mathrm{cm}^{2}\right)$, the electrons among plasmas take swift quiver, by which the adsorbed energy by electrons is converted to thermal energy of the random thermal motional plasma [10]. The self-radiation of plasma can bring out X-ray, electrons, ions, and neutrons, but there is still lack of deep understanding on the specific mechanism for the EMP generation. To further study the EMP features, a Faraday cup was developed [11].

This work aims to evaluate the EMP emissions from an ICF integrated diagnostic system when a long pulse (ns) laser of several $\mathrm{kJ}$ level is utilized to ignite the target and it is expected to provide possible solutions to electromagnetic interference (EMI) problems and also lay a preliminary foundation to achieve fusion. The microwave can potentially damage various interior electrics including energy balance components, X-ray cameras, and optical parts.

\section{Experiment}

All the measurements were performed outside a 9-beam Nd-glass laser facility as sketched in Fig. 1, where various diagnostic setups were installed inside and outside a 2.7-meter diameter sphere chamber. The 9-beam laser included 8-beam laser from the northern hemisphere (4 beams) and the southern hemisphere (4 beams), plus a backlight beam normally from top of the north-south line 45 degrees. The detailed information on laser energy, focal spot size, backlight energy, and laser pulse width used in this measurement is listed in Table1, in which an estimated peak power intensity is around $10^{15} \mathrm{~W} / \mathrm{cm}^{2}$, and the amplitude of emitted microwave are compared and analyzed as functions of these parameters. The solid targets used in this study were typical dual-media targets of Rayleigh-Taylor instability. The chamber vacuum remained less than $5 \times 10^{-3} \mathrm{~Pa}$.

Table 1 Detailed information of laser used to illuminate solid targets.

\begin{tabular}{ccccc}
\hline & $\begin{array}{c}\text { Laser energy } \\
(\mathrm{kJ})\end{array}$ & $\begin{array}{c}\text { Focal spot } \\
\text { size }(\mu \mathrm{m})\end{array}$ & $\begin{array}{c}\text { Back ground } \\
\text { light }(\mathrm{kJ})\end{array}$ & $\begin{array}{c}\text { Laser pulse } \\
\text { width }(\mathrm{ns})\end{array}$ \\
\hline $\mathrm{L1}^{\#}$ & 5.6 & 500 & 1 & 3 \\
$\mathrm{~L}^{\#}$ & 0 & 500 & 1 & 3 \\
$\mathrm{~L}^{\#}$ & 0 & 500 & 2.7 & 3 \\
L4 $^{\#}$ & 3.35 & 500 & 0 & $1(0.2)$ \\
L5 $^{\#}$ & 3.2 & 500 & 2.7 & $1(3)$ \\
L6 $^{\#}$ & 5.6 & 500 & 1.8 & 2 \\
L7 $^{\#}$ & 5.6 & 500 & 2.6 & 2 \\
\hline
\end{tabular}

A conical antenna was designed and made from copper coated nickel to collect the EMP signals, linked by a standard 50-coaxial cable to a high-speed real-time oscilloscope as shown in Fig. 1. The antenna was placed close to a glassy flange fixed in the chamber wall to reduce effects from the 
hardware. The antenna had been both calibrated and modeled from $0.8 \mathrm{GHz}$ to $5 \mathrm{GHz}$, by which the peak gain of antenna in the frequency ranges of $1 \mathrm{GHz}$ to $5 \mathrm{GHz}$ and distribution of electrical field obtained at $1 \mathrm{GHz}$ are displayed in Fig. 2.

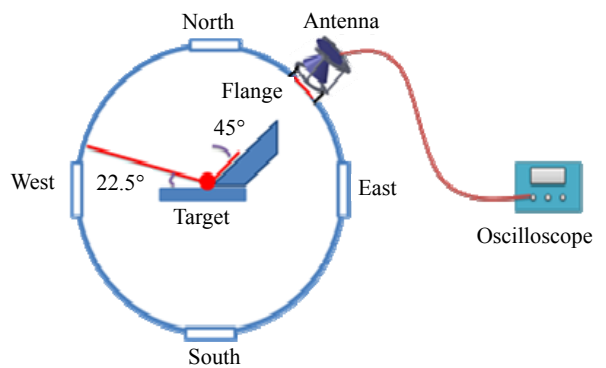

Fig. 1 Schematic representation of the experimental setup.

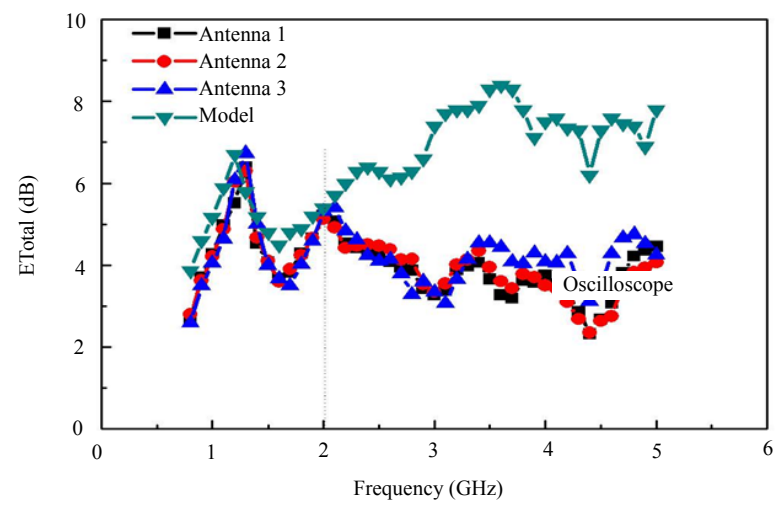

(a)

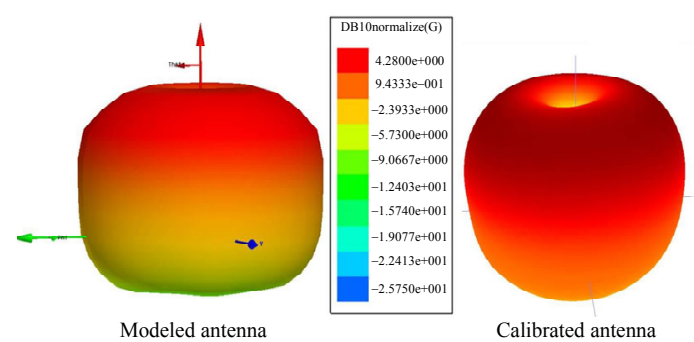

(b)

Fig. 2 Parameters for antenna used: (a) peak gains of the used antenna at different frequencies obtained by calibration and simulation and (b) calibrated and simulated electric field distributions along the antenna at $1 \mathrm{GHz}$.

The transfer function $\mathbf{H}(\omega)$ is of importance because it bridges the voltage signal and electromagnetic field, which was obtained by simulating the antenna. The effects of measurement system on low-frequency domains caused non-negligible noises. In order to eliminate this noise, Tikhonov regularization with L-curve analysis was selected to address the distorted signals. The measured voltage $\mathbf{V}(\omega)$ can be related to the electrical field $\mathbf{E}(\omega)$ by a transfer function of $\mathbf{H}(\omega)$ as expressed by (1), in equal to a time-domain formulation in (2), which can be mathematically discrete into $\mathbf{V}=\mathbf{H E}$, where $\mathbf{V}$ and $\mathbf{E}$ denote $m$-directional vector and

$$
\begin{gathered}
\mathbf{V}(\omega)=\mathbf{H}(\omega) \mathbf{E}(\omega) \\
\mathbf{V}(t)=\mathbf{H}(t) \mathbf{E}(t)
\end{gathered}
$$

where $\mathbf{H}$ is an $m \times m$ matrix. We then use the zero order of Tikhonov regularization to figure out the extremum $\min _{e \in R^{n}}\left(\|H e-v\|^{2}+\mu\|e\|^{2}\right)$, where $\|\cdot\|$ denotes the Euclidean norm, and $\mu$ is a parameter that needs to be cleared. The solution to the above problem is

$$
e_{\mu, i}=H_{i}^{*} v_{i} / H_{i}^{*} H_{i}-\mu, \quad i=1,2, \cdots, m .
$$

In this case, the $\mathrm{L}$ curve analysis is employed to confirm the parameter $\mu$, and then the electrical field can be obtained by reversely undergoing these procedures.

\section{Results and Discussion}

Figure 3 presents some typical voltage wave forms that have been recorded and labeled from $\mathrm{L}^{\#}$ to $\mathrm{L}^{\#}$ with amplitudes ranging from $1.5 \mathrm{~V}$ to $6.6 \mathrm{~V}$. Note that the measurements are all conducted outside the chamber, which means the resultant EMP would be greatly reduced when it penetrates the thick quartz glass (to ensure good leak-tightness), making the signals not that strong. Each EMP has transient duration of up to $80 \mathrm{~ns}$ before the signal attenuation decays into noise.

However, all the wave forms show that there are some unfixable periods for EMP peaks, where oscillation of the waveforms can be observed with time course, and the time intervals of some evident peaks are assessed from several ns to dozens of ns. The wave oscillation lasts nearly $20 \mathrm{~ns}$ without any decaying with regard to $\mathrm{L}^{\#}$, which implies there are at least two coupled modes of different frequencies when 8-beams laser and the backlight energy are totally accounted to $6.6 \mathrm{~kJ}(5.6 \mathrm{~kJ}+1 \mathrm{~kJ})$. 


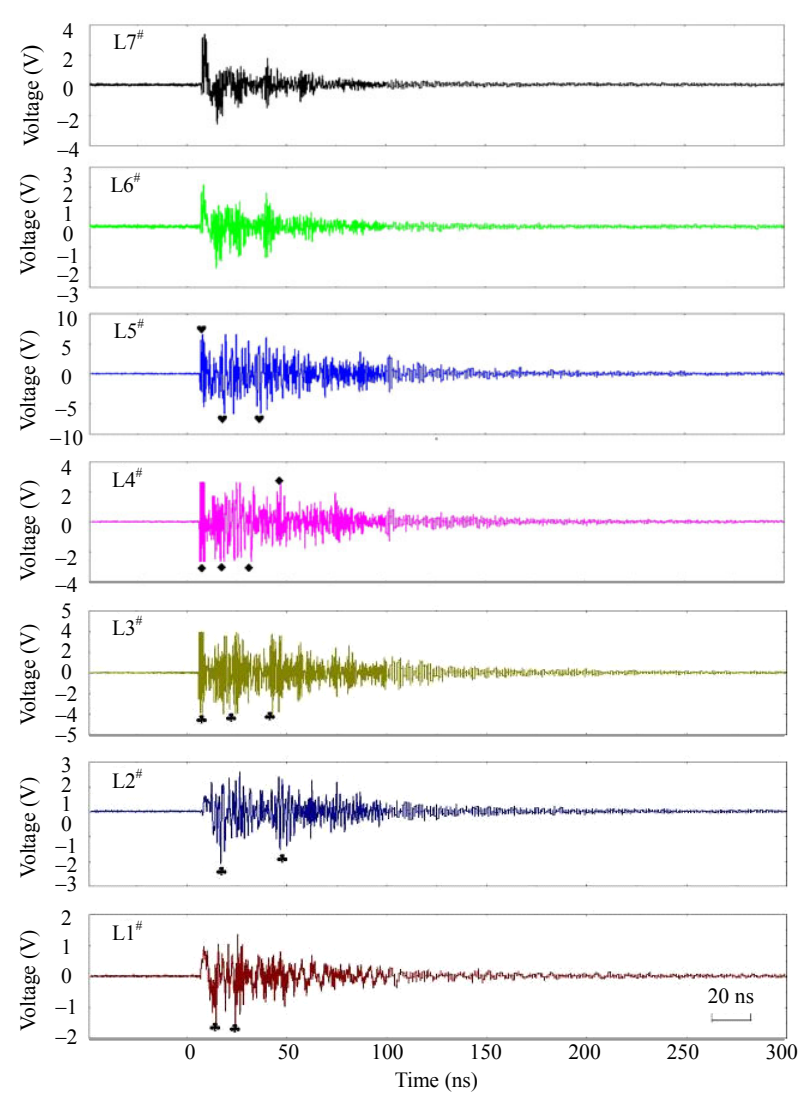

Fig. 3 Typical coupled voltage outputs recorded by the cone antenna at varying laser input and laser width.

To emphasize the effects of backlight laser beam, $\mathrm{L}^{\#}$ is carried out by adjusting the backlight to be $1 \mathrm{~kJ}$ and leaving the 8-beams laser. There are two separate peaks at $15 \mathrm{~ns}(2.1 \mathrm{~V})$ and $50 \mathrm{~ns}(1.5 \mathrm{~V})$, which are much bigger than that of $\mathrm{L}^{\#}$. To increase the backlight to $2.7 \mathrm{~kJ}$ as depicted in $\mathrm{L}^{\#}$, the peak voltage is enhanced to $4.0 \mathrm{~V}$, and the couple modes of at least four different frequencies occur in this process, indicating the backlight shot can not only determine the microwave intensity, but also create more different radiating modes.

On the other hand, the effects of laser width on the formation of electromagnetic impulse are taken into account. With respect to $\mathrm{L}^{\#}$, we use 4-beams $1 \mathrm{~ns}$ pulses $(3.2 \mathrm{~kJ})$ and 1 -beam $0.2 \mathrm{~ns}$ pluses $(0.15 \mathrm{~kJ})$ to react with the solid target. Several peaks with amplitudes of $2.6 \mathrm{~V}$ are obtained, and a series of strong oscillations are caused by the shorter pluses lasting for about $40 \mathrm{~ns}$ and then decayed into noise. We maintain the $1 \mathrm{~ns}$ pulses and meanwhile add
$2.7 \mathrm{~kJ}$ backlight laser ( $3 \mathrm{~ns}$ ) in $\mathrm{L}^{\#}$ to see the changes. A pronounced peak of $6.1 \mathrm{~V}$ is achieved, and three evident modes are coupled during this process, suggesting that the backlight shot could be related to the total radiation energy. The resultant waveforms in $\mathrm{L}^{\#}$ and $\mathrm{L}^{\#}$ are carried out to further confirm the conclusion that the intensity of backlight beam is critical to the strength of microwave.

As shown in $\mathrm{L}^{\#}(4.0 \mathrm{~V})$ and $\mathrm{L}^{\#}(6.1 \mathrm{~V})$, an increase in total energy from the 8-beams laser with shorter pulse tends to induce extra radiation upon the reactions between laser and target, differing to the situation in $\mathrm{L}^{\#}$ and $\mathrm{L}^{\#}$, where the 9-beams laser has identical width (3 ns) and aroused similar voltage amplitudes. The corresponding frequency wave profiles obtained by conducting the fast frontier transform (FFT) are shown in Fig. 4, from which it can be seen that the frequency bandwidth resides at around $2 \mathrm{GHz}$ or lower. It is also noted that the emissions have characteristic peak structures with several peaks and all the peaks located below approximately $2 \mathrm{GHz}$ possibly due to the glass flange act as an attenuator or filer, which is more evident when the antenna is placed outside the metal shell as measured in this study (the highest voltage peak was only $0.4 \mathrm{~V}$ ).

The corresponding electric field (E-field) strength was obtained by combining the antenna simulation and mathematical method as depicted in Fig. 5. E-field signals reached from over $200 \mathrm{~V} / \mathrm{m}$ to $600 \mathrm{~V} / \mathrm{m}$, which indicates some appropriate shielding methods are particularly required to protect various electronics including the charged coupled device (CCD), dilation X-ray imager (DIXI), and the filter-fluorescer experiment. The results in this experiment are able to provide a suitable standard on how to design additional shielding for all neighboring diagnostic instruments since the sources of EMP cannot be completely eliminated, even we tried the best to control or lower the EMP origins. 


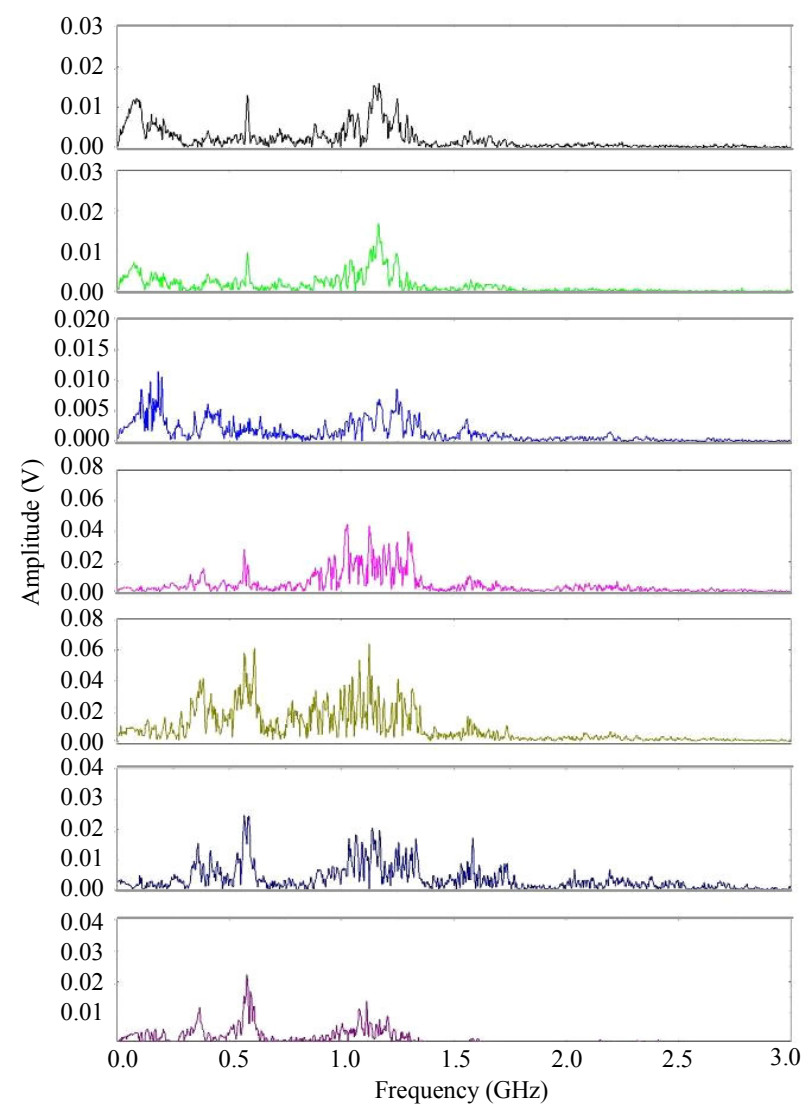

Fig. 4 Fast Fourier transform (FFT) of the corresponding voltage signals shown in Fig. 3.

The patterns of EMP transportation are possibly responsible for the coupled voltage output in this measurement, which mainly stem from the laser-target interaction and diagnostic-generated EMI [12], where some preliminary results of EMI induced by various diagnostic setups are evaluated. More tests needed to be elaborated and performed to confirm the specific origins of EMI since the testing environment is extremely complicated.

To understand the multiple peaks in this study, the EMP generated from laser-target interaction randomly propagates inside the chamber as sketched in Fig. 6. There are probably four different coupled modes that are manifested in all testing results. The first peak may come from direct laser-target interaction labeled by the arrow as shown in Fig. 6(a) because of the largest intensity and the earliest time arriving at the antenna. In this case, the propagating length before hitting the antenna equals approximately to the radius of this sphere chamber
$(1.35 \mathrm{~m})$. Assuming the speed of microwave inside the chamber (vacuum) is the light speed, the diameter corresponds to $9 \mathrm{~ns}$.

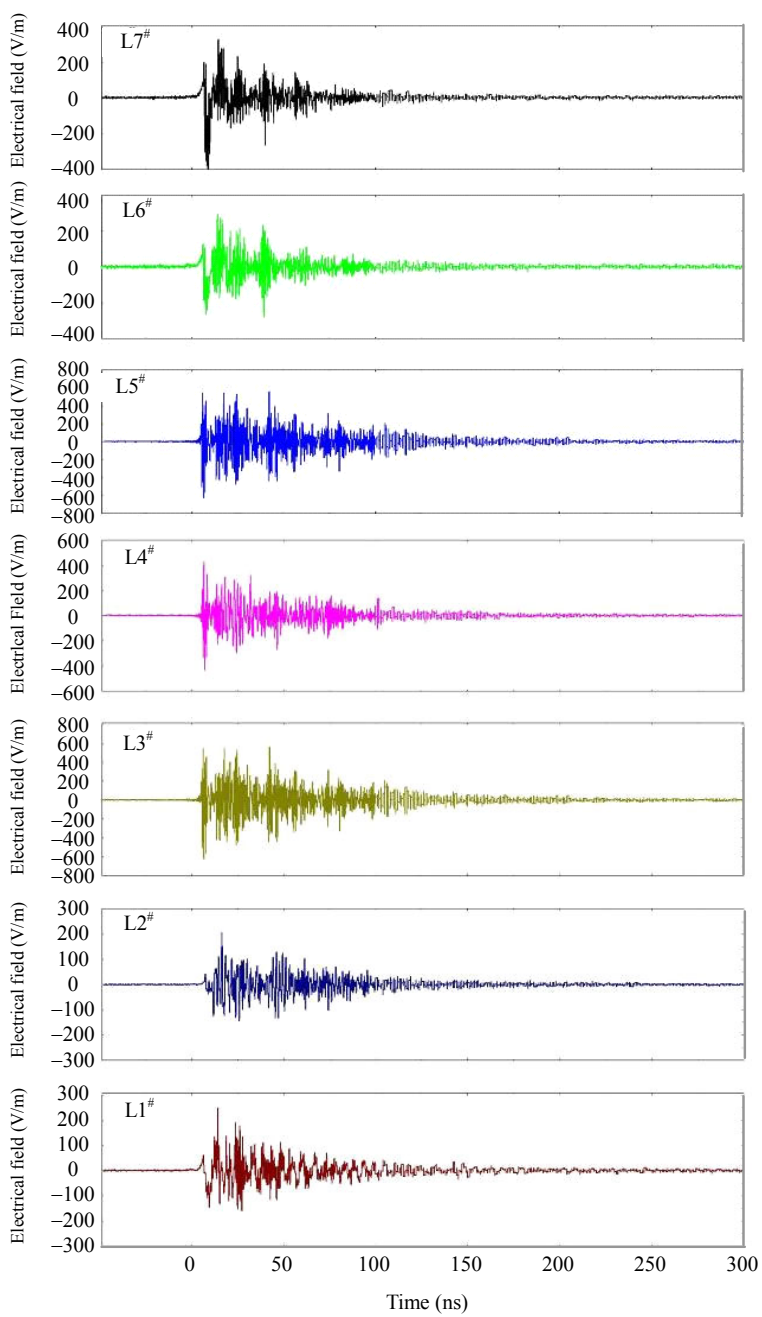

Fig. 5 Electric field strength obtained by processing voltage signal data [with $\mathbf{E}(\omega)=\mathbf{V}(\omega) / \mathbf{H}(\omega)]$.

For another thing, the microwave is originated from reflection of inner wall as represented by the arrows. The second peak in $\mathrm{L}_{2}^{\#}$ waveform delays over $20 \mathrm{~ns}$ (6meters), which could be attributed to several times of reflections as depicted in Fig. 6(b). Note that the second peak in $\mathrm{L}_{2}^{\#}$ is weaker than the first one, which is mainly due to energy loss caused by the chamber wall adsorption and scattering. The effects of interior diagnostic instruments are also taken into account when their electric components are very sensitive to electromagnetic pulses. Since the radiated electric can also generate huge EMP, it is expected to further intensify the reflected waves 
as demonstrated in Fig. 6(c). Some typical waveforms including $\mathrm{L}^{\#}, \mathrm{~L}^{\#}$, and $\mathrm{L}^{\#}$ can be

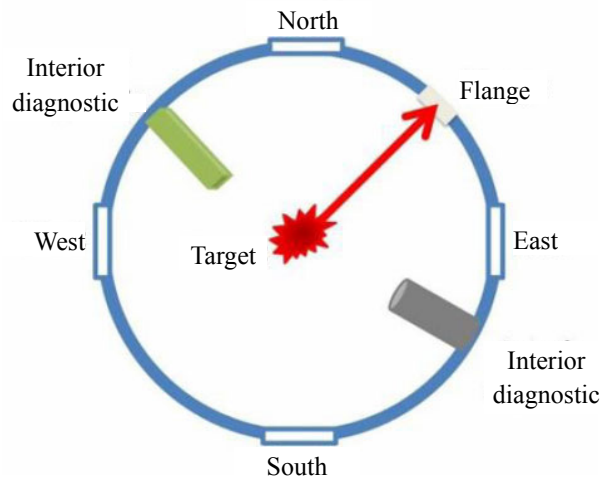

(a)

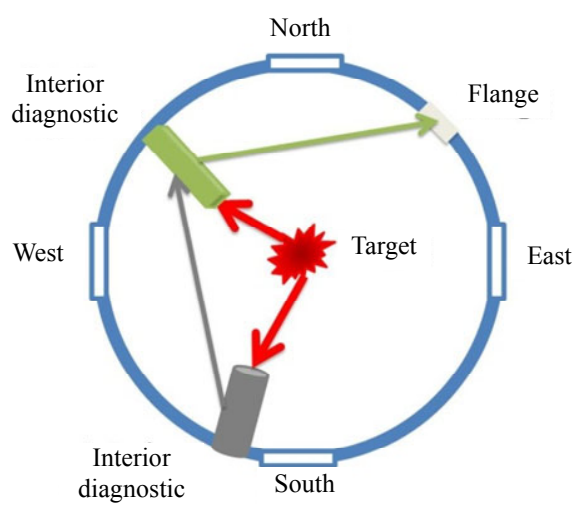

(c) presumably explained by this coupled mode as they all have multiple peaks of similar amplitudes.

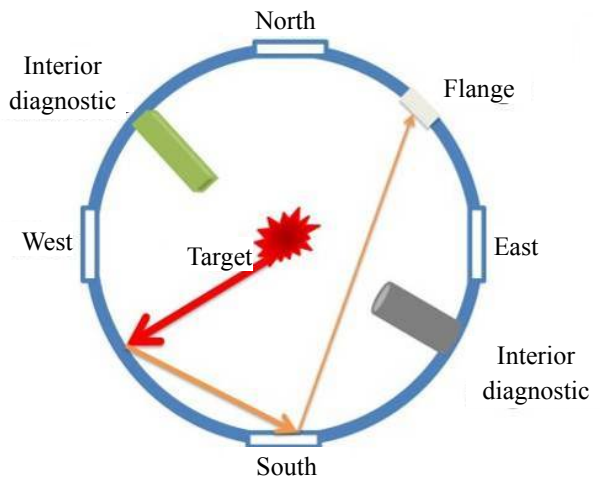

(b)

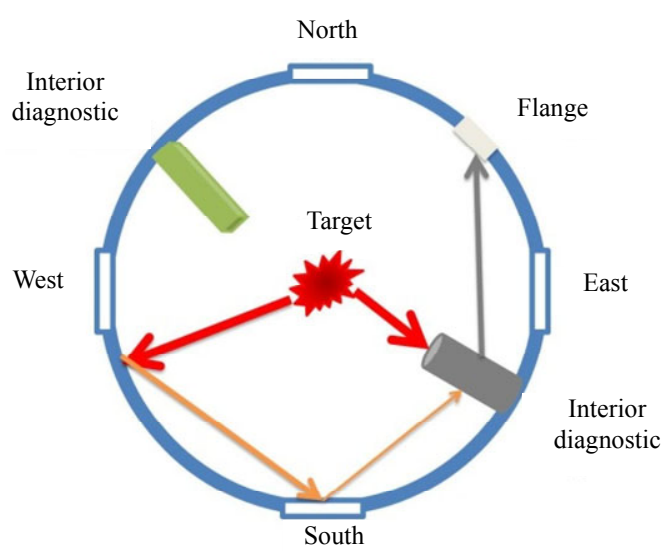

(d)

Fig. 6 Mechanism for EMP generation with a multiple peaks: (a) modes for EMP induced by a high-power laser directly interacting with solid target, (b) reflection from the inner chamber wall, (c) radiations from the interior diagnostics, and (d) adsorption and scattering of the wall in combination with inside electric-generated.

Attentions should be paid to some peaks with time intervals of $10 \mathrm{~ns}$ to $20 \mathrm{~ns}$ (equals to $3-\mathrm{m}$ to $6-\mathrm{m}$ length) owing to the inside diameter of sphere chamber being totally $2.7 \mathrm{~m}$, meaning that the possible mode in Fig. 6(d) can maintain the wave strength and appear in a delayed way, in which multiple scattering (long distance transmission) on the inner wall surface interacts with some interior electronics to preserve or improve the intensity $\left(\mathrm{L1}^{\#}\right)$ to a high level.

To understand the relationship between the EMP amplitude and laser energy, it should relate the entry laser to escaping electron plasma density, which can be expressed in (3), where $\lambda$ is the laser wavelength and $m_{e}$ is the electron mass.

$$
n_{c}=\frac{m_{e}}{4 \pi e^{2}}\left(\frac{2 \pi c}{\lambda}\right)^{2} .
$$

It can be seen that the electron number is closely pertinent to the laser wavelength. When a beam of backlight laser is added onto target surface, the escaping electrons must be increased and the spatial distribution can also be multiplied by characterizing the components that electrons strike [7], leading to the electrons escape in multi-directional way.

\section{Conclusions}

EMP generated by some powerful lasers interacting with solid targets was measured by using 
a cone antenna. The amplitudes of EMP were analyzed as functions of laser energy and bandwidth. All the waveforms in this experiment had multiple peaks and possessed duration of dozens of ns before they attenuated into noise. Several coupled modes were established to interpret these specific temporal electric signal oscillograms, indicating that the EMP was not only stemming from direct laser-target interaction, but also was induced by the interior diagnostics by inner wall reflection.

\section{Acknowledgment}

We would like to thank Dr. Peng Wang at University of Electric Science and Technology of China (UESTC) and Dr. Ziyu Chen at China Academy of Engineering Physics for their help in processing the data and understanding the potential mechanisms. This work was financially supported by the Fundamental Research Funds for the Central Universities (No. ZYGX2015J108) and National Natural Science Foundation of China (Nos. 11575166 and 5158 1140).

Open Access This article is distributed under the terms of the Creative Commons Attribution 4.0 International License (http://creativecommons.org/licenses/by/4.0/), which permits unrestricted use, distribution, and reproduction in any medium, provided you give appropriate credit to the original author(s) and the source, provide a link to the Creative Commons license, and indicate if changes were made.

\section{References}

[1] R. Qiu, Y. Y. Liu, W. Q. Li, Y. X. Pan, S. L. Wang, Q. $\mathrm{Li}$, et al., "Measurement and validation of the cross section in the FLUKA code for the production of $\mathrm{Zn}-63$ and $\mathrm{Zn}-65$ in $\mathrm{Cu}$ targets for low-energy proton accelerators," Nuclear Science and Techniques, 2014, 25(1): 6-10.

[2] T. M. Tzschentke and W. J. Schmidt, "Ignition on the national ignition facility: a path towards inertial fusion energy," Nuclear Fusion, 2009, 49(10): 593-598.

[3] R. Kodama, P. A. Norreys, K. Mima, A. E. Dangor, R. G. Evans, H. Fujita, et al., "Fast heating of ultrahigh-density plasma as a step towards laser fusion ignition," Nature, 2001, 412(6849): 798-800.

[4] M. J. Mead, D. Neely, J. Gauoin, R. Heathcote, and P. Patel, "Electromagnetic pulse generation within a petawatt laser target chamber," Review of Scientific Instruments, 2004, 75(10): 4225-4227.

[5] C. G. Brown, E. Bond, T. Clancy, S. Dangi, D. C. Eder, W. Ferguson, et al., "Assessment and mitigation of electromagnetic pulse (EMP) impacts at short-pulse laser facilities," Journal of Physics: Conference Series, 2010, 244(3): 681-687.

[6] H. Hora, J. B. Heinrich, F. P. Boody, R. Höpfl, K. Jungwirth, B. Králikova, et al., "Effects of ps and ns laser pulses for giant ion source," Optics Communications, 2002, 207(1-6): 333-308.

[7] C. G. Brown, A. Throop, D. Eder, and J. Kimbrough, "Electromagnetic pulses at short-pulse laser facilities," Journal of Physics: Conference Series, 2008, 112(3): 1231-1236.

[8] Z. Y. Chen, J. F. Li, J. Li, and Q. X. Peng, "Microwave radiation mechanism in a pulse-laser-irradiated $\mathrm{Cu}$ foil target revisited," Physica Scripta, 2011, 83(5): 434-440.

[9] S. Varma, J. Spicer, B. Brawley, and J. Miragliotta, "Plasma enhancement of femtosecond laser-induced electromagnetic pulses at metal and dielectric surfaces," Optical Engineering, 2014, 53(5): 688-696.

[10] S. Eliezer, The interaction of high-power lasers with plasmas. Boca Raton: CRC Press, 2002.

[11] J. Prokupek, J. Kaufman, D. Margarone, M. Krůs, A. Velyhan, J. Krása, et al., "Development and first experimental tests of Faraday cup array," Review of Scientific Instruments, 2014, 85(1): 013302-1-013302-6.

[12] C. G. Brown, J. Ayers, B. Felker, W. Ferguson, J. P. Holder, S. R. Nagel, et al. "Assessment and mitigation of diagnostic-generated electromagnetic interference at the National Ignition Facility," Review of Scientific Instruments, 2012, 83(10): 10D729-1-10D729-3. 